\title{
Study of mutagenic effects of a packaging system with oxygen absorbers on sweet bakery products
}

\author{
R. A. Sueiro, M. J. Garrido \& M. Araujo \\ Lab. Microbiology (Instituto de Investigación y Análisis Alimentarios), \\ Universidad de Santiago de Compostela, Spain
}

\begin{abstract}
In the present study, the potential mutagenic effects of a packaging system for food containing oxygen absorbers was evaluated on a popular sweet bakery product (glazed doughnuts) stored at $20^{\circ} \mathrm{C}$ along its extended self life by using the Salmonella typhimurium His test with the tester strains TA98, TA100, TA1535 and TA1537 and Escherichia coli Trp'test with strains WP2uvrA and WP2uvrA pKM101. All samples were assayed in the presence and absence of an exogenous metabolizing system (S9 from rat liver). During the study, 3 lots were prepared and analysed: lot 1 consisted of control samples without absorber sachets. In lot 2 one oxygen absorber sachet was added into the package and in lot 3 two oxygen absorbers were used. All samples were packaged in high barrier pouches and stored at $20^{\circ} \mathrm{C}$ along the shelf life of the product, determined in other study as 4 days for control samples and 8 days for samples packaged with oxygen absorbers. As it was expected, the active concept itself did not show mutagenic activity. In addition, the control samples and samples in contact with the oxygen scavengers were also negatives in all sampling times (at days 0, 5 and 10). These results support the safe use of this type of active packaging to extend the shelf life of commercial doughnuts because the interactions between the bakery product and the package system did not represent a risk for the consumer health under the conditions tested.
\end{abstract}

Keywords: active packaging system, oxygen absorbers, sweet bakery products, S. Typhimurium his-test, E. coli Trp-test. 


\section{Introduction}

Most bakery products stored at room temperature have a limited shelf-life. Between the main causes of deterioration are included oxidative degradation processes, changes in the content and distribution of water and microbial spoilage (Galić et al. [1]). Prolonging the shelf life of baked goods and pastries for alternatives ways to the use of additives, can be accomplished through the use of active packaging. Active packaging is an innovative concept that encompasses a collection of technologies in response to specific problems to improve the maintenance and extend the shelf life of foods (Vermeiren et al. [2]). Its use not only has the advantage of improving the conditions under which the food is supplied to the consumer, but it may also mean a lower cost of production and distribution as well as increased capacity of companies expansion since it has the potential ability to prolong the shelf life of packaged foodstuffs.

Due to the high potential of this technology, it has been developed and marketed a variety of concepts for use in active packaging of foods such as oxygen absorbers, carbon dioxide absorbers, ethanol emitters, ethylene absorbers and moisture absorbers (Rooney [3]). Oxygen absorbers are among the most suitable concepts for use in bakery products (Rooney [3]; Galić et al. [1]). They are often readily oxidizable chemicals commercialized in small pockets of oxygen permeable material that it is introduced into passive or conventional packaging with the food (Galić et al. [1]). Active packaging systems should not release their constituents if they are intended merely to absorb undesirable substances from the packaged foodstuff, such as oxygen. However, sachets containing active substances are often in contact with packaged foods, giving rise to the possibility that their migration into the foodstuff might be significant, especially in the case of moist, fatty and/or acid foodstuffs (López-Cervantes et al. [4]).

The European Union (EU), sensitive to many undesirable interactions that it can occur between the packaging and packaged foods, has developed the Framework Regulation (EC) no 1935/2004 [5] on materials and articles intended to come into contact with food where the active food contact materials were included in its field of application. Moreover, Regulation (EC) no 450/2009 [6] is a specific measure that lays down rules for active materials and articles to be applied in addition to the general requirements established in Regulation (EC) no $1935 / 2004$ for their safe use. A communitary list of authorised substances shall be established after the European Safety Authority (EFSA) has performed a risk assessment on each substance including their toxicological properties (EFSA [7]) but to date it has not been published. With regard to materials and articles intended to come into contact with food, it has recently published the Commission Regulation (EC) no 10/2011 [8]. This latter Regulation supposes an update of applicable rules to such plastic materials which includes the list of authorized monomers and additives in the EU.

Despite numerous efforts by the EU to regulate the materials that come into contact with food, recent studies with other plastic materials authorized in the EU, such as epoxy resins based in bisphenol A, have shown that components of 
this kind of resins and their derived products react with food components giving rise to formation of new and unknown compounds not subject to European legislation (Petersen et al. [9]; Coulier et al. [10]). Since oxygen absorber sachets are often in contact with packaged food, it cannot rule out the formation of new compounds when the passive plastic packaging, the active concept and the food interact. It is therefore necessary to check that their use does not represent a health risk for consumers under a toxicological point of view (Hotchkiss [11].

Considering the absence of information available about that, the aim of the present study was to evaluate the potential mutagenic effects of a high barrier packaging system for food containing oxygen absorber sachets on a popular sweet bakery product (glazed doughnuts) stored at $20^{\circ} \mathrm{C}$ along its extended self life using the Salmonella His $^{-}$test and Escherichia coli trp ${ }^{-}$test. The mutagenic effects of oxygen absorbers used in the present study were also evaluated with the same bacterial test systems. Both assays are between the most internationally agreed testing methods to identify and characterise potential hazard of new and existing chemical substances and chemical mixtures and they are used in regulatory safety testing (Organisation for Economic Co-operation and Development [12]). The assays are based on the ability of substances to induce point mutations in bacterial strains following DNA damage.

\section{Materials and methods}

\subsection{Experimental conditions assayed}

Doughnuts freshly prepared by a local manufacturer were packaged into polypropylene biaxially oriented film coated with acrylic and polyvinylidene chloride resin authorized for use in contact with foodstuff. Before sealing the packages, they were divided into 3 lots. In Lot 1 no active concept was incorporated within. In Lot 2 was added one iron-based oxygen-absorber sachet in each package and in lot 3 were added two absorber oxygen sachets. All lots were sampled at days 0,5 and 10 in order to assess the mutagenic potential of packaged doughnuts. All samples were kept at $20^{\circ} \mathrm{C}$ during the study to simulate the conditions in which they are commercialized. Moreover, it was evaluated the mutagenic activity of oxygen absorbers used in the experiments. Lots from each sample type were collected each sampling day and they were submitted to extraction with dichloromethane-methanol $(2: 1, \mathrm{v} / \mathrm{v})$. The organic extracts were dried over anhydrous sodium sulphate and concentrated to dryness under vacuum. Before performing the assays, they were dissolved in dimethyl sulfoxide (DMSO).

\subsection{Mutagenicity assays}

Sample extracts obtained from doughnuts and oxygen absorbers were tested using the plate incorporation method as described by Maron and Ames [13] for $S$. typhimurium and by Urios et al. [14] for E. coli. For experiments with S. typhimurium strains, the top agar was supplemented with $10 \mathrm{ml}$ of $0.5 \mathrm{mM}$ 
histidine/biotin solution per $100 \mathrm{ml}$ agar, and mutations to histidine independence were scored on minimal glucose agar plates (Maron and Ames [13]). For experiments with E. coli strains, mutations to tryptophan independence were scored on minimal glucose agar plates supplemented with $0.5 \mathrm{mg}$ tryptophan per litre (Urios et al. [14]).

The S9 microsomal fraction from the livers of male Sprague-Dawley rats pretreated with a phenobarbital-methylcholanthrene mixture was purchased from Iffa Credo (L'Arbesle, France) and used as the external metabolic activation system. Both types of sample extracts were tested in the presence and absence of a mixture of $10 \%(\mathrm{v} / \mathrm{v}) \mathrm{S} 9$ fraction in the S9 mix, which serves to convert mutagenic compounds in need of metabolic activation to their active mutagenic form. The concentrations of cofactors in the $\mathrm{S} 9 \mathrm{mix}$ (before adding them to the overlay) were $4 \mathrm{mM}$ NADP, $5 \mathrm{mM}$ glucose-6-phosphate, $33 \mathrm{mM} \mathrm{KCl}, 8 \mathrm{mM}$ $\mathrm{MgCl}_{2}$ and $100 \mathrm{mM}$ sodium phosphate buffer (pH 7.4) (Maron and Ames [13]).

At least two complete assays were performed for each sample extract. All experiments were carried out in triplicate using a minimum of five doses. Positive and negative controls were included in each assay. Data from representative assays for the oxygen absorber extracts with the test doses evaluated are shown in Results and Discussion section where representative data for the doughnuts extracts along the period of study are also shown.

The statistical analysis of the data was based on biological mechanistic models proposed by Margolin et al. [15]. The SALM program (Kim and Margolin [16]) was used to fit Margolin's models to the data and select the model with the greatest likelihood. The Goodness of fit test calculated for each data set allows us to know if the data followed the fitted model. The estimated slope of the initial linear region (no. of revertants/mg sample equivalent) was used as a measure of mutagenic potency and for determining mutagenicity via a significance test. In this way, a positive response was obtained when the mutagenicity test p-value was less than 0.05 . A sample was deemed to be mutagenic if at least one strain/activation combination yielded a reproducible positive response.

\section{Results and discussion}

Tables 1-3 present the mutagenic potential of the solvent extractable compounds recovered from oxygen absorber samples using $S$. typhimurium His ${ }^{-}$and $E$. coli $\mathrm{Trp}^{-}$tests in one independent mutagenicity assay. The dose levels assayed were between 0.313 and $5 \mathrm{mg}$ sample equivalent/plate in all cases.

It can be seen the absence of mutagenic activity obtained with $S$. typhimurium strains TA98, TA100, TA1535 and TA1537 (Tables 1 and 2) either in the presence or absence of external metabolic activation. These results are indicative that oxygen absorber extracts did not induce frameshift mutations (detected with strains TA98 y TA1537), but neither have the ability to induce base pairs substitutions primarily affecting G-C base-pairs (detected with strains TA100 and TA1535) in the test system employed. No toxicity was evident at the highest dose assayed ( $5 \mathrm{mg} /$ plate). Negative results were also obtained when E. coli 
Table 1: $\quad$ Mutagenicity of oxygen absorber extracts in S. typhimurium strains TA98 and TA100.

\begin{tabular}{|c|c|c|c|c|}
\hline \multirow{3}{*}{ Dose (mg/plate) } & \multicolumn{4}{|c|}{ Number of re vertan ts per plate } \\
\hline & \multicolumn{2}{|c|}{ TA98 } & \multicolumn{2}{|c|}{ TA100 } \\
\hline & $-\mathrm{S} 9$ & $10 \% \mathrm{~S} 9$ & $-\mathrm{S} 9$ & $10 \% \mathrm{~S} 9$ \\
\hline DMSO control & $25 \pm 5,3$ & $29 \pm 2.5$ & $129 \pm 7.4$ & $121 \pm 11.3$ \\
\hline 5 & $26 \pm 4.4$ & $36 \pm 6.4$ & $137 \pm 4.2$ & $124 \pm 8.4$ \\
\hline 2.5 & $35 \pm 9.1$ & $29 \pm 3.1$ & $141 \pm 10.2$ & $123 \pm 10.1$ \\
\hline 1.25 & $30 \pm 4.6$ & $31 \pm 4.5$ & $142 \pm 6.7$ & $126 \pm 6.1$ \\
\hline 0.625 & $25 \pm 4.0$ & $33 \pm 3.2$ & $139 \pm 5.9$ & $126 \pm 7.8$ \\
\hline 0,313 & $25 \pm 3.1$ & $31 \pm 4.6$ & $150 \pm 7.9$ & $124 \pm 4.4$ \\
\hline Slope $^{1}$ & 0.60 & 0.87 & 0.00 & 0.04 \\
\hline $\mathrm{H}_{2} \mathrm{O}$ control & - & - & $124 \pm 11.0$ & - \\
\hline Positive control $1^{2}$ & $226 \pm 17.9$ & $201 \pm 14.4$ & $450 \pm 12.7$ & $305 \pm 17.0$ \\
\hline
\end{tabular}

Values are means \pm SD of triplicate plates of one representative experiment. -, not tested. Revertants/mg of sample equivalent. ${ }^{2}$ Positive controls: TA98/-S9 (2,4,7-trinitro-9-fluorenone, $0.01 \mu \mathrm{g} / \mathrm{plate}$; TA100/-S9 (sodium azide, $1 \mu \mathrm{g} / \mathrm{plate}$ ), TA98 and TA100/4\% S9 (1 and $2.5 \mu \mathrm{g} / \mathrm{plate}$ 2-aminofluorene, respectively).

Table 2: $\quad$ Mutagenicity of oxygen absorber extracts in S. typhimurium strains TA1535 and TA1537.

\begin{tabular}{|c|c|c|c|c|}
\hline \multirow{3}{*}{ Dose (mg/plate) } & \multicolumn{4}{|c|}{ Number of revertants per plate } \\
\hline & \multicolumn{2}{|c|}{ TA1537 } & \multicolumn{2}{|c|}{ TA1535 } \\
\hline & $-\mathrm{S} 9$ & $10 \% \mathrm{~S} 9$ & $-\mathrm{S} 9$ & $10 \% \mathrm{~S} 9$ \\
\hline DMSO control & $10 \pm 1.5$ & $16 \pm 3.5$ & $9 \pm 2.1$ & $16 \pm 1.5$ \\
\hline 5 & $12 \pm 2.0$ & $17 \pm 3.1$ & $11 \pm 3.2$ & $19 \pm 3.2$ \\
\hline 2.5 & $10 \pm 2.1$ & $16 \pm 4.0$ & $12 \pm 2.7$ & $17 \pm 2.7$ \\
\hline 1.25 & $13 \pm 0.6$ & $16 \pm 4.0$ & $11 \pm 2.7$ & $16 \pm 2.7$ \\
\hline 0.625 & $13 \pm 2.1$ & $17 \pm 3.1$ & $10 \pm 2.5$ & $18 \pm 2.0$ \\
\hline 0,313 & $11 \pm 2.0$ & $18 \pm 3.0$ & $10 \pm 1.7$ & $18 \pm 2.5$ \\
\hline Slope $^{1}$ & 0.05 & 0.04 & 0.33 & 0.31 \\
\hline $\mathrm{H}_{2} \mathrm{O}$ control & - & - & $12 \pm 2.5$ & - \\
\hline Positive control $^{2}$ & $182 \pm 14.3$ & $90 \pm 6.0$ & $272 \pm 8.5$ & $181 \pm 8.5$ \\
\hline
\end{tabular}

Values are means \pm SD of triplicate plates of one representative experiment. -, not tested. Revertants/mg of sample equivalent. ${ }^{2}$ Positive controls: TA1537/-S9 (9-aminoacridine, $50 \mu \mathrm{g} /$ plate; TA1535/-S9 (sodium azide, $0.5 \mu \mathrm{g} / \mathrm{plate}$ ), TA1537 and TA1535/10\% S9 (50 $\mu \mathrm{g} / \mathrm{plate}$ 2-aminoanthracene, respectively). 
strains WP2uvrA and WP2uvrA pKM101 were used in the assay at the same doses tested (Table 3), either with or without S9 fraction in the S9 mix. The latter data are indicative that active concept extracts analysed not induce base-pair substitutions affecting A-T base-pairs. Therefore, the results suggest that the use of such active concept itself does not represent a genotoxic hazard under the test conditions employed.

Table 3: Mutagenicity of oxygen absorber extracts in E. coli strains WP2uvrA and WP2uvrA pKM101.

\begin{tabular}{|c|c|c|c|c|}
\hline \multirow{3}{*}{ Dose (mg/plate) } & \multicolumn{4}{|c|}{ Number of re vertan ts per plate } \\
\hline & \multicolumn{2}{|c|}{ WP2uvrA } & \multicolumn{2}{|c|}{ WP2uvrA pKM101 } \\
\hline & $-\mathrm{S} 9$ & $10 \% \mathrm{~S} 9$ & $-\mathrm{S} 9$ & $10 \% \mathrm{~S} 9$ \\
\hline DMSO control & $36 \pm 5.7$ & $37 \pm 4.0$ & $98 \pm 4.6$ & $105 \pm 12.7$ \\
\hline 5 & $37 \pm 4.0$ & $40 \pm 6.7$ & $103 \pm 5.0$ & $105 \pm 12.5$ \\
\hline 2.5 & $44 \pm 4.2$ & $38 \pm 1.0$ & $105 \pm 8.4$ & $116 \pm 6.7$ \\
\hline 1.25 & $38 \pm 7.8$ & $45 \pm 4.6$ & $101 \pm 4.4$ & $108 \pm 8.6$ \\
\hline 0.625 & $44 \pm 4.0$ & $47 \pm 7.6$ & $107 \pm 5.3$ & $112 \pm 6.7$ \\
\hline 0,313 & $41 \pm 10.2$ & $43 \pm 5.3$ & $101 \pm 2.5$ & $111 \pm 5.0$ \\
\hline Slope $^{1}$ & 0.00 & 0.00 & 0.52 & 0.00 \\
\hline $\mathrm{H}_{2} \mathrm{O}$ control & - & - & $122 \pm 4.5$ & - \\
\hline Positive control $^{2}$ & $178 \pm 12.0$ & $196 \pm 13.4$ & $449 \pm 12.7$ & $672 \pm 21.0$ \\
\hline
\end{tabular}

Values are means $\pm \mathrm{SD}$ of triplicate plates of one representative experiment. -, not tested. Revertants/mg of sample equivalent. ${ }^{2}$ Positive controls: WP2uvrA and IC3327/-S9 (methyl methanesulfonate, $100 \mu \mathrm{g} /$ plate; WP2uvrA and WP2uvrA pKM101/4\% S9 (10 and $0.5 \mu \mathrm{g} / \mathrm{plate}$ 2-aminoanthracene, respectively).

These results could be expected considering the chemical composition provided by the supplier, which indicate that the oxygen absorber sachets used in the present study contained iron powder covered with sea salt and natural zeolith impregnated with a sodium chloride solution. The iron under appropriate humidity conditions uses up residual oxygen to form nontoxic iron oxide (Smith et al. [17]). Moreover, studies performed to know the behaviour of two commercial sachets of iron-based oxygen-absorber systems with respect to migration of active ingredients into foodstuffs showed that sodium chloride and iron were the compounds that migrate from the oxygen-scavenging system into solid foods (López-Cervantes et al. [4]). Therefore, it could be expected that these compounds interact with the packaged foodstuff along with those migrating from the plastic package.

To rule out the possible mutagenicity of doughnuts packaged with this active packaging system, mutagenicity assays of the product were performed along its extended self life. The doughnuts self lives were evaluated in other study as 4 days for control samples packaged without oxygen absorbers and 8 days for samples packaged with the active concepts based on sensorial and microbiological analyses (data not shown). The mutagenic potential of doughnut 
extracts was also evaluated using $S$. typhimurium His $^{-}$and E. coli Trp ${ }^{-}$tests. The five dose levels assayed for these sample extracts were between 1.25 and $20 \mathrm{mg}$ sample equivalent/plate in all cases. Representative results of this part of the study are reported in the Figures 1-3 as reversion rates, which represent the total number of revertant colonies at a dose of $20 \mathrm{mg}$ of doughnut extract per plate divided by the number of revertant colonies appearing for the negative DMSO control. Under this treatment of data, ratios greater than the numerical value of two are indicative of a mutagenic response. However, to determine if each sample extract was or not considered mutagenic the five doses tested were subjected to statistical analysis by using the SALM program, as indicated in Materials and Methods section.

Figure 1 show the reversion rate obtained with doughnut extracts along the period of study when S. typhimurium strain TA98 was used in the assay. Neither the control (lot 1) nor the bakery product packaged with one and two oxygen absorber sachets (lot 2 and lot 3, respectively) reached ratios greater than the numerical value of two, both with and without the addition of S9 fraction in the assay. The statistical analysis of the data obtained during the assays along the period studied confirmed the absence of mutagenic activity in all sample extracts analysed. No toxicity was evident at the higher doses tested, which was assayed at the maximum tolerated dose based upon its solubility limit in DMSO. In the same way, Figures 2 and 3 present data of reversion rates obtained for the sample extracts with strains TA100 and WP2uvrA pKM101, respectively. Again, it is shown that in no case the value of two was reached. Negative results were also obtained with TA1535, TA1537 y WP2uvrA (data not shown).

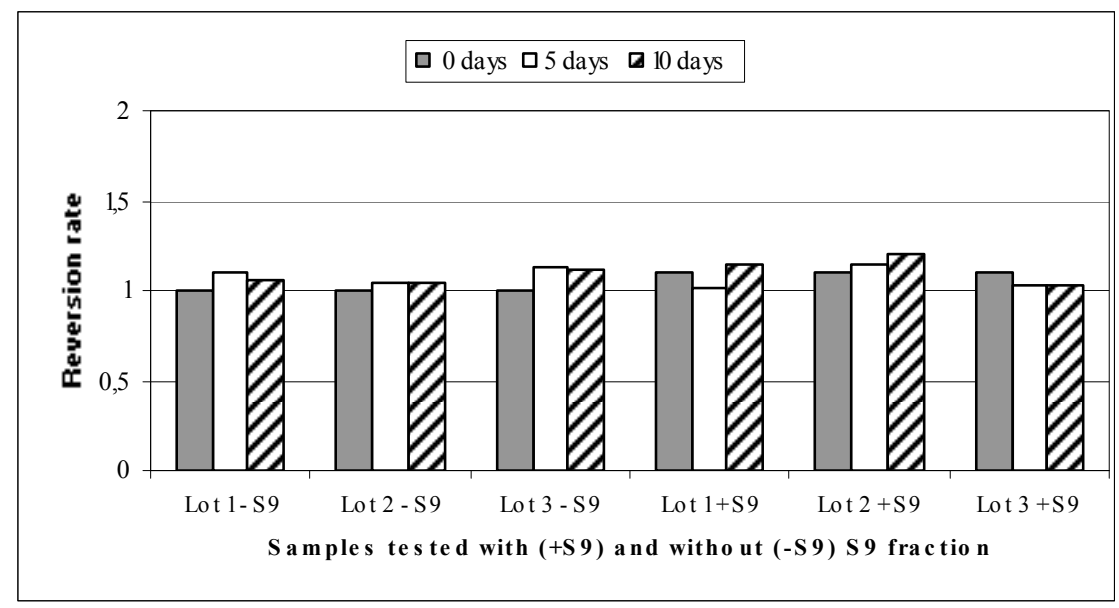

Figure 1: Reversion rates obtained for the doughnuts extracts in the mutagenicity assay with S. typhimurium strain TA98 in the presence and absence of S9 fraction at the higher dose assayed (20 mg/plate). Lot 1: doughnuts packaged without oxygen absorbers. Lot 2 and lot 3: doughnuts packaged with one and two oxygen absorbers, respectively. 


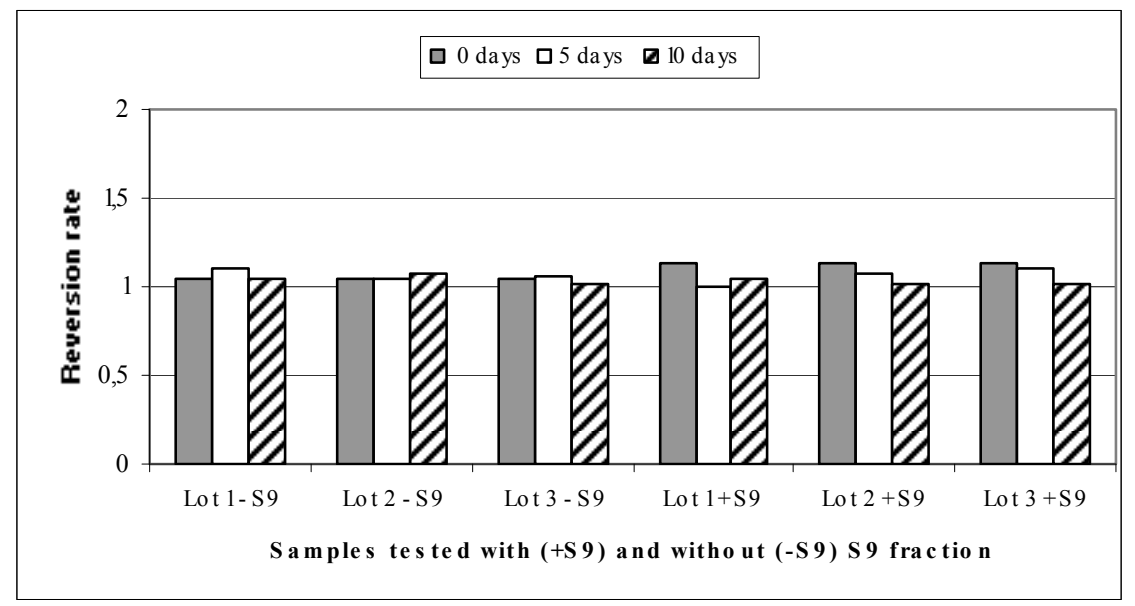

Figure 2: Reversion rates obtained for the doughnuts extracts in the mutagenicity assay with S. typhimurium strain TA100 in the presence and absence of S9 fraction at the higher dose assayed (20 $\mathrm{mg} / \mathrm{plate})$. Lot 1: doughnuts packaged without oxygen absorbers. Lot 2 and lot 3: doughnuts packaged with one and two oxygen absorbers, respectively.

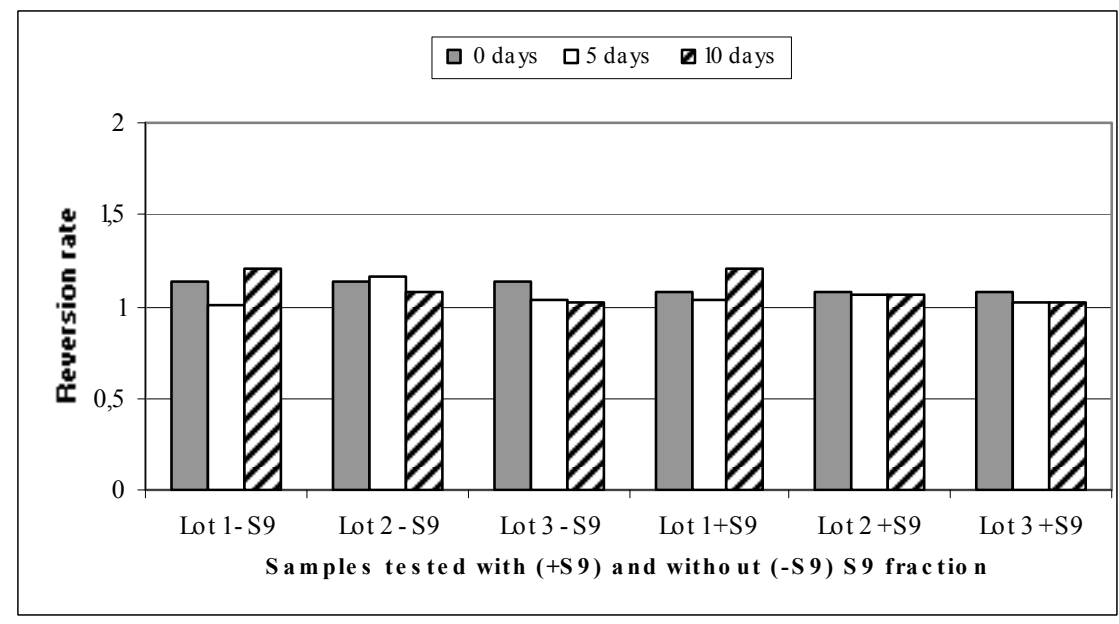

Figure 3: Reversion rates obtained for the doughnuts extracts in the mutagenicity assay with E. coli strain WP2uvrA pKM101 in the presence and absence of S9 fraction at the higher dose assayed (20 $\mathrm{mg} / \mathrm{plate})$. Lot 1: doughnuts packaged without oxygen absorbers. Lot 2 and lot 3: doughnuts packaged with one and two oxygen absorbers, respectively. 
It can also highlight the lack of differences between the results obtained in the doughnuts packaged without oxygen absorbers (lot 1) and the use of one (lot 2) or two (lot 3) absorbers. These results support the safe use of this type of active packaging to extend the shelf life of commercial doughnuts because the interactions between the bakery product and the active packaged system (including the plastic package) did not represent a risk for the consumer health under the conditions tested. We consider that an important extension of the present work would include an examination for genotoxicity of these extracts in mammalian assay systems covering different end-points.

\section{Acknowledgement}

This work was partially funded by la Xunta de Galicia (PGIDT99PXI23701B and PGIDT00INN08E).

\section{References}

[1] Galić, K., Ćurić, D., \& Gabrić, D. Shelf life of packaging bakery goods. A review. Critical Reviews in Food Science and Nutrition. 49, pp. 405-426, 2009.

[2] Vermeiren, L., Devlieghere, F., van Beest, M., de Kruijf, N. \& Debevere, J. Developments in the active packaging of foods. Trends Food Sci. Technol. 10, pp. 77-86, 1999.

[3] Rooney, M.L Active packaging in polymer films. Active Food Packaging, edited by M.L. Rooney (London: Blackie), pp. 74-110, 1995.

[4] López-Cervantes, J., Sánchez-Machado, D.I. Pastorelli, S., Rijk, R. \& Paseiro-Losada, P. Evaluating the migration of ingredients from active packaging and development of dedicated methods: a study of two ironbased oxygen absorbers. Food Addit. Contam. 20, pp. 291-299, 2003.

[5] Regulation (EC) No 1935/2004 of the European Parliament and of the Council of 27 October 2004 on materials and article intended to come into contact with food and repealing Directives 80/590/EEC and 89/109/EEC. Official J. L 338, pp. 4-17, 2004.

[6] Regulation (EC) No 450/2009 on active and intelligent materials and articles intended to come into contact with food. Official J. L 135, pp. 3-11, 2009.

[7] European Food Safety Authority (EFSA). Guidelines on submission of a dossier for safety evaluation by the EFSA of active or intelligent substances present in active and intelligent materials and articles intended to come into contact with food. The EFSA Journal. 1208, pp. 1-11, 2009.

[8] Commission Regulation (EU) No 10/2011 on plastic materials and articles intended to come into contact with food. Official J. L 12, pp. 1-89, 2011.

[9] Petersen, H., Biereichel, A., Burseg, K., Simat, T.J. \& Steinhart, H. Bisphenol A diglycidyl ether (BADGE) migrating from packaging materials "disappears" in food: reaction with food components. Food Addit. Contam. 25, pp. 911-920, 2008. 
[10] Coulier, L., Bradley, E.L., Bas, R.C., Verhoeckx, K.C.M., Driffield, M., Harmer, N. \& Castle, L. Analysis of reaction products of food contaminants and ingredients: Bisphenol A Diglycidyl Ether (BADGE) in canned foods. J. Agric. Food Chem., 58, pp. 4873-4882, 2010.

[11] Hotchkiss, J.H. Food-packaging interactions influencing quality and safety. Food Addit. Contam. 14, pp. 601-607, 1997.

[12] Organisation for Economic Co-operation and Development. OECD Guidelines for the testing of chemicals, section 4: human effects. Test No 471: Bacterial mutation test. Available in http://www.oecdilibrary.org/environment/test-no-471-bacterial-reverse-mutationtest 9789264071247 -en.

[13] Maron, D.M. \& Ames, B.N. Revised methods for the Salmonella mutagenicity test. Mutat. Res. 113, pp. 173-215, 1983.

[14] Urios, A., Herrera, G. \& Blanco, M. Detection of oxidative mutagens in strains of Escherichia coli deficient in the OxyR or Mut $\mathrm{Y}$ functions: dependence on SOS mutagenesis. Mutat. Res. 332, pp. 9-15, 1995.

[15] Margolin, B.H. Kaplan, N. \& Zeiger, E. Statistical analysis of the Ames Salmonella/microsome test. Proc. Natl. Acad. Sci. U.S.A. 78, pp. 37793783, 1981.

[16] Kim, B.S. \& Margolin, B.H. Statistical methods for the Ames Salmonella assay: a review. Mutat. Res. 436, pp. 113-122, 1999.

[17] Smith, J. P., Ramaswamy, H. S. \& Simpson, B. K. (1990). Developments in food packaging technology. Trends Food Sci. Technol. 1:112-119. 